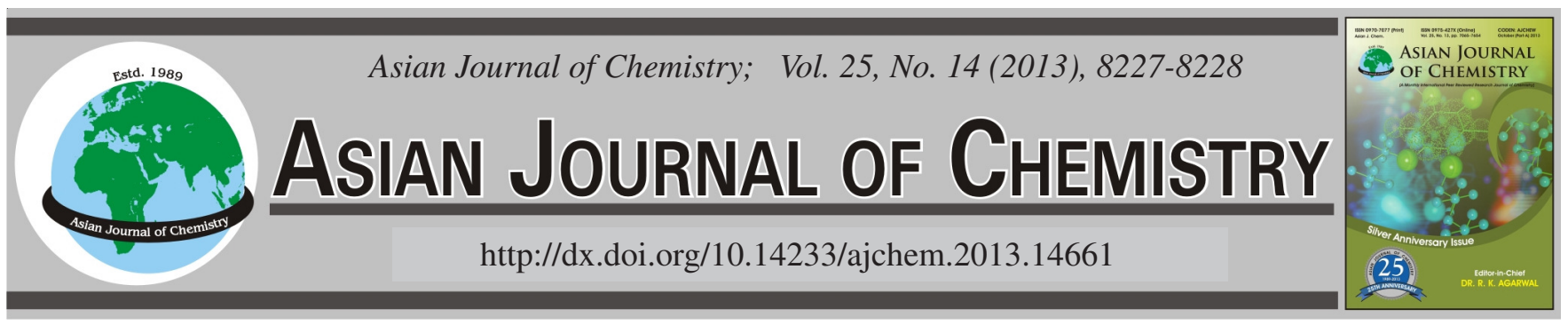

NOTE

\title{
Study on Novel Structure of Molybdenum Tungsten Imidazole Complex: $\left(\mathrm{H}_{12} \mathrm{MoW}_{7} \mathrm{O}_{30}\right)\left(\mathrm{C}_{3} \mathrm{H}_{4} \mathrm{~N}_{2}\right)_{4}\left(\mathrm{H}_{2} \mathrm{O}\right)_{3}$
}

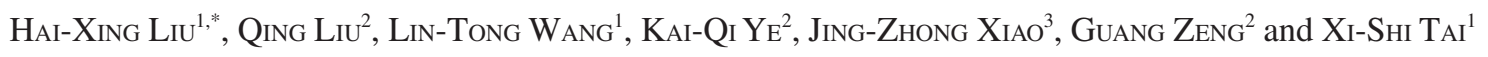

${ }^{1}$ Chemistry and Chemical and Environmental Engineering College, Weifang University, Weifang 261061, P.R. China

${ }^{2}$ State Key Laboratory of Inorganic Synthesis and Preparative Chemistry, College of Chemistry, Jilin University, Changchun 130012, P.R. China ${ }^{3}$ CEMDRX, Department of Physics, University of Coimbra, Coimbra 3004-516, Portugal

*Corresponding author: E-mail: haixingliu@tom.com

(Received: 9 October 2012;

Accepted: 12 August 2013)

AJC-13916

\begin{abstract}
A novel molybdenum tungsten imidazole complex $\left(\mathrm{H}_{12} \mathrm{MoW}_{7} \mathrm{O}_{30}\right)\left(\mathrm{C}_{3} \mathrm{H}_{4} \mathrm{~N}_{2}\right)_{4}\left(\mathrm{H}_{2} \mathrm{O}\right)_{3}$ with the m.f. $\mathrm{C}_{12} \mathrm{H}_{34} \mathrm{MoN}_{8} \mathrm{O}_{33} \mathrm{~W}_{7}$ has been synthesized from a single solution reaction and the crystal structure has been determined by means of single-crystal X-ray diffraction. The Mo atom is coordinated by seven $\mathrm{O}$ atoms. There are two kinds coordinations mode for $\mathrm{W}$ atom. The crystal is stabilized by $\mathrm{O}-\mathrm{H} \cdots \mathrm{O}$ and $\mathrm{N}-\mathrm{H} \cdots \mathrm{O}$ hydrogen bonds interaction.
\end{abstract}

Key Words: Molybdenum tungsten complex, Imidazole, Structure analysis.

Supramolecular compounds based on polyoxometalates construct a small subset of the large family of polyoxometalates which have attracted considerable attention due to variety of their structures and potential application ${ }^{1-3}$. The self-assembly of supramolecular structure performs via ligand-metal coordination bonds, hydrogen bonds, electrostatic force and $\pi-\pi$ stacking interaction ${ }^{4}$. Recently, the strategy of self-assembly through intermolecular weak interactions is a focus of the design of new polyoxometalates based hybrids ${ }^{5}$, which have been intensively investigated in many important aspects such as catalysis, electrical conductivity, magnetic properties and biological chemistry ${ }^{6}$. In this paper, the novel molybdenum tungsten imidazole complex is reported.

All commercially obtained reagent-grade chemicals were used without further purification. A mixture of $\mathrm{MnSO}_{4}(0.01$ mmol, $0.002 \mathrm{~g}),\left(\mathrm{NH}_{4}\right)_{2} \mathrm{WO}_{4}(0.1 \mathrm{mmol}, 0.03 \mathrm{~g}),\left(\mathrm{NH}_{4}\right)_{2} \mathrm{MoO}_{4}$ $(0.1 \mathrm{mmol}, 0.02 \mathrm{~g})$, imidazole $(0.1 \mathrm{mmol}, 0.007 \mathrm{~g})$, citric acid $(0.1 \mathrm{mmol}, 0.02 \mathrm{~g})$ and dilute $\mathrm{HCl}$ were added into $20 \mathrm{~mL}$ water with $20 \%(\mathrm{v} / \mathrm{v})$ ethanol and heated for $5 \mathrm{~h}$ at $343 \mathrm{~K}$. The solution was obtained by filtration after cooling the reaction to room temperature. Colourless block single crystals suitable for $\mathrm{X}$-ray measurements were obtained after a few weeks.

The title crystal structure (Fig. 1) is built up of molybdenum tungsten acid, imidazole and water molecular. The 1D chain structure extending along $b$ axis is shown in Fig. 2. The View of the three-fold interpenetration network along the $b$ axis is shown in Fig. 3. The crystal data and structure refinement is shown in Table-1. The Mo atom is coordinated by seven $\mathrm{O}$ atoms (one $\mathrm{O} 1$, two $\mathrm{O} 1 \mathrm{w}$, two $\mathrm{O} 2 \mathrm{w}$, two $\mathrm{O} 3 \mathrm{w}$ ). The $\mathrm{W} 2$ atom is coordinated by five $\mathrm{O}$ atoms and the others $\mathrm{W}$ atoms are coordinated by six $\mathrm{O}$ atoms. The $\mu 2-\mathrm{O} 1$ atom bridges Mo1 and $\mathrm{W} 2$. The $\mu 3-\mathrm{O} 8$ atom bridges $\mathrm{W} 1, \mathrm{~W} 3$ and $\mathrm{W} 4$. The $\mu 4$ $\mathrm{O} 7$ atom bridges two $\mathrm{W} 1, \mathrm{~W} 2$ and $\mathrm{W} 4$, while $\mu 4-\mathrm{O} 13$ atom bridges two W3, W4 and W5. The distances of d(Mo-O) and $\mathrm{d}(\mathrm{W}-\mathrm{O})$ are in the range of 2.428-2.496 $\AA$ and 1.697-2.506 $\AA$, respectively. The bond angles of Mo1-O1-W2 is $161.2^{\circ}$. Selected bond lengths and bond angles are shown in Table- 2 .

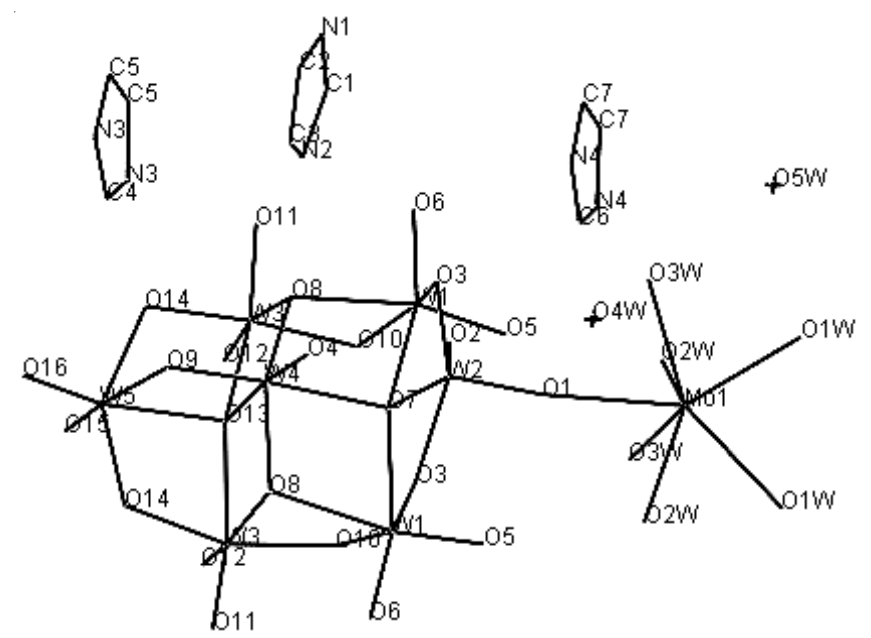

Fig. 1. Molecular structure of the title complex 


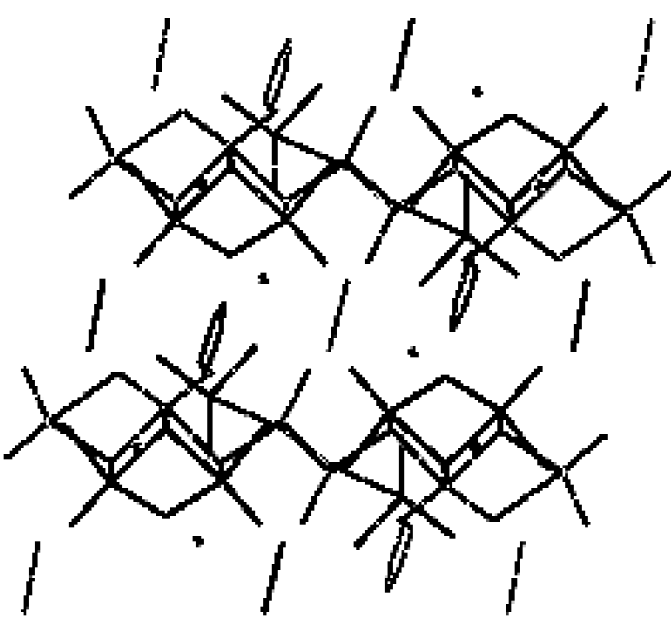

Fig. 2. 1D chain structure extending along $b$ axis

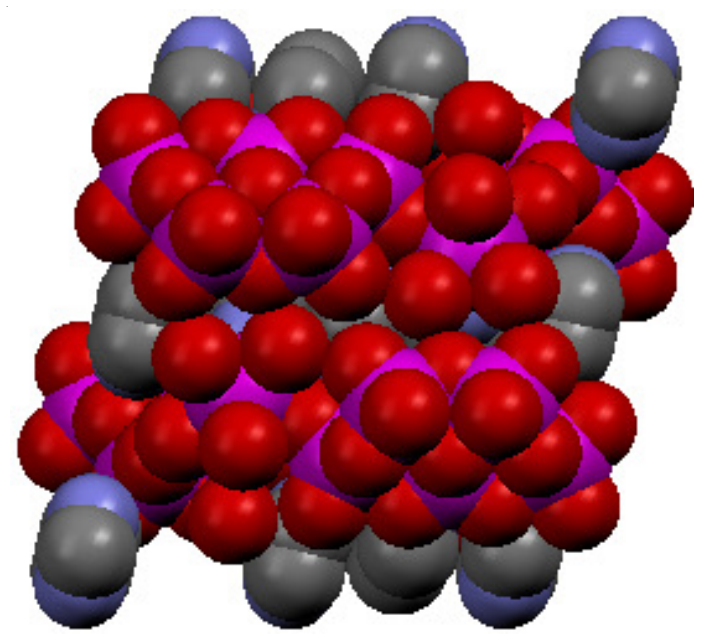

Fig. 3. Perspective view of the 3D packing diagram along the $b$ axis

\begin{tabular}{|c|c|}
\hline \multicolumn{2}{|c|}{$\begin{array}{c}\text { TABLE-1 } \\
\text { CRYSTAL DATA AND STRUCTURE REFINEMENT } \\
\text { FOR THE TITLE COMPLEX }\end{array}$} \\
\hline Empirical formula & $\mathrm{C}_{12} \mathrm{H}_{34} \mathrm{MoN}_{8} \mathrm{O}_{33} \mathrm{~W}_{7}$ \\
\hline Formula weight & 2201.36 \\
\hline Temperature & 291(2) K \\
\hline Wavelength & $0.71073 \AA$ \\
\hline Crystal system, space group & Monoclinic, $\mathrm{C}^{2} / \mathrm{m}$ \\
\hline Unit cell dimensions & $a=15.6529(8) \AA \quad \alpha=90^{\circ}$ \\
\hline & $\mathrm{b}=19.3356(8) \AA \beta=103.975(4)^{\circ}$ \\
\hline & $c=14.1045(5) \AA \gamma=90^{\circ}$ \\
\hline Volume & $4142.5(3) \AA^{3}$ \\
\hline Z, Calculated density & $4,3.530 \mathrm{Mg} / \mathrm{m}^{3}$ \\
\hline Absorption coefficient & $19.755 \mathrm{~mm}^{-1}$ \\
\hline $\mathrm{F}(000)$ & 3944 \\
\hline Crystal size & $0.28 \times 0.24 \times 0.22 \mathrm{~mm}$ \\
\hline Theta range for data collection & 1.49 to $26.00^{\circ}$ \\
\hline Limiting indices & $\begin{array}{l}-8<=\mathrm{h}<=19,-23<=\mathrm{k}<=22,-17 \\
<=1<=17\end{array}$ \\
\hline Reflections collected/unique & $12725 / 4205[\mathrm{R}(\mathrm{int})=0.0141]$ \\
\hline Completeness to $\theta=26$ & $99.8 \%$ \\
\hline Absorption correction & Semi-empirical from equivalents \\
\hline Max and min transmission & 0.02 and 0.01 \\
\hline Refinement method & Full-matrix least-squares on $\mathrm{F}^{2}$ \\
\hline Data/restraints/parameters & $4205 / 0 / 296$ \\
\hline Goodness-of-fit on $\mathrm{F}^{2}$ & 1.092 \\
\hline Final $R$ indices $[I>2 \sigma(I)]$ & $\mathrm{R} 1=0.0210, \mathrm{wR} 2=0.0653$ \\
\hline $\mathrm{R}$ indices (all data) & $\mathrm{R} 1=0.0237, w \mathrm{R} 2=0.0676$ \\
\hline Largest diff. peak and hole & 0.618 and -1.868 e. $\AA^{-3}$ \\
\hline
\end{tabular}

TABLE-2

SELECT BOND LENGTHS [̊̊] AND ANGLES $\left.{ }^{\circ}\right]$ FOR THE TITLE COMPLEX

\begin{tabular}{|c|c|c|c|}
\hline $\mathrm{C}(1)-\mathrm{N}(1)$ & $1.310(8)$ & $\mathrm{O}(4 \mathrm{~W})-\mathrm{H}(4 \mathrm{X})$ & 0.8500 \\
\hline $\mathrm{C}(1)-\mathrm{N}(2)$ & $1.366(7)$ & $\mathrm{W}(1)-\mathrm{W}(2)$ & 3.1993(3) \\
\hline $\mathrm{C}(1)-\mathrm{H}(1)$ & 0.9300 & $\mathrm{~N}(1)-\mathrm{C}(1)-\mathrm{N}(2)$ & $108.0(5)$ \\
\hline $\mathrm{C}(2)-\mathrm{C}(3)$ & $1.307(9)$ & $\mathrm{C}(3)-\mathrm{C}(2)-\mathrm{N}(1)$ & $107.8(5)$ \\
\hline $\mathrm{C}(2)-\mathrm{N}(1)$ & $1.377(9)$ & $\mathrm{C}(2)-\mathrm{C}(3)-\mathrm{N}(2)$ & $109.0(6)$ \\
\hline $\mathrm{C}(2)-\mathrm{H}(2)$ & 0.9300 & $\mathrm{~N}(2)-\mathrm{C}(3)-\mathrm{H}(3)$ & 125.5 \\
\hline $\mathrm{C}(3)-\mathrm{N}(2)$ & $1.343(8)$ & $\mathrm{N}(4)-\mathrm{C}(6)-\mathrm{H}(6)$ & 123.9 \\
\hline $\mathrm{C}(4)-\mathrm{N}(3)$ & $1.290(7)$ & $\mathrm{C}(1)-\mathrm{N}(1)-\mathrm{C}(2)$ & $107.8(5)$ \\
\hline $\mathrm{C}(5)-\mathrm{N}(3)$ & $1.372(7)$ & $\mathrm{C}(4)-\mathrm{N}(3)-\mathrm{C}(5)$ & $108.5(5)$ \\
\hline $\mathrm{C}(6)-\mathrm{N}(4)$ & $1.286(8)$ & $\mathrm{C}(7)-\mathrm{N}(4)-\mathrm{H}(4 \mathrm{~A})$ & 127.3 \\
\hline $\mathrm{C}(7)-\mathrm{N}(4)$ & $1.375(9)$ & $\mathrm{W}(2)-\mathrm{O}(1)-\mathrm{Mo}(1)$ & $161.2(3)$ \\
\hline $\mathrm{Mo}(1)-\mathrm{O}(3 \mathrm{~W})$ & $2.428(4)$ & $\mathrm{W}(4)-\mathrm{O}(4)-\mathrm{W}(2)$ & $105.1(2)$ \\
\hline $\mathrm{Mo}(1)-\mathrm{O}(1)$ & $2.470(5)$ & $\mathrm{W}(4)-\mathrm{O}(9)-\mathrm{W}(5)$ & $108.9(2)$ \\
\hline $\operatorname{Mo}(1)-\mathrm{O}(1 \mathrm{~W})$ & $2.470(4)$ & $\mathrm{O}(8)-\mathrm{W}(1)-\mathrm{W}(2)$ & $87.34(8)$ \\
\hline $\mathrm{Mo}(1)-\mathrm{O}(2 \mathrm{~W})$ & $2.496(4)$ & $\mathrm{O}(1)-\mathrm{W}(2)-\mathrm{O}(2)$ & $105.5(2)$ \\
\hline $\mathrm{N}(2)-\mathrm{H}(2 \mathrm{~A})$ & 0.8600 & $\mathrm{O}(2)-\mathrm{W}(2)-\mathrm{O}(7)$ & $150.6(2)$ \\
\hline $\mathrm{O}(1)-\mathrm{W}(2)$ & $1.704(5)$ & $\mathrm{O}(2)-\mathrm{W}(2)-\mathrm{W}(1)$ & $135.66(5)$ \\
\hline $\mathrm{O}(2)-\mathrm{W}(2)$ & $1.756(5)$ & $\mathrm{O}(7)-\mathrm{W}(2)-\mathrm{W}(1)$ & $42.25(3)$ \\
\hline $\mathrm{O}(3)-\mathrm{W}(1)$ & $1.941(4)$ & $\mathrm{O}(4)-\mathrm{W}(2)-\mathrm{W}(1)$ & $83.32(8)$ \\
\hline $\mathrm{O}(4)-\mathrm{W}(4)$ & $1.732(5)$ & $\mathrm{O}(9)-\mathrm{W}(4)-\mathrm{O}(4)$ & $105.9(2)$ \\
\hline $\mathrm{O}(5)-\mathrm{W}(1)$ & $1.707(4)$ & $\mathrm{O}(4)-\mathrm{W}(4)-\mathrm{O}(13)$ & $173.0(2)$ \\
\hline $\mathrm{O}(6)-\mathrm{W}(1)$ & $1.697(4)$ & $\mathrm{O}(4)-\mathrm{W}(4)-\mathrm{O}(7)$ & $83.6(2)$ \\
\hline $\mathrm{O}(7)-\mathrm{W}(2)$ & $2.141(5)$ & $\mathrm{O}(15)-\mathrm{W}(5)-\mathrm{O}(16)$ & $105.0(2)$ \\
\hline $\mathrm{O}(8)-\mathrm{W}(4)$ & $1.894(3)$ & $\mathrm{O}(15)-\mathrm{W}(5)-\mathrm{O}(13)$ & $106.1(2)$ \\
\hline $\mathrm{O}(9)-\mathrm{W}(4)$ & $1.723(5)$ & $\mathrm{O}(15)-\mathrm{W}(5)-\mathrm{O}(9)$ & $174.7(2)$ \\
\hline $\mathrm{O}(1 \mathrm{~W})-\mathrm{H}(1 \mathrm{X})$ & 0.9600 & $\mathrm{O}(16)-\mathrm{W}(5)-\mathrm{O}(9)$ & $80.3(2)$ \\
\hline
\end{tabular}

\section{ACKNOWLEDGEMENTS}

This study were supported by the Natural Science Foundation of Shandong Province (No. ZR2010BL025), State Key Laboratory of Inorganic Synthesis and Preparative Chemistry (Jilin University) (No. 2011-13), MOE Key Laboratory of Analytical Chemistry for Life Science (Nanjing University) (No. KLACLS1002) and the National Science Foundation of China (No. 21171132) and Open Project of State Key Laboratory of Supramolecular Structure and Materials, Jilin University (No. sklssm201323).

\section{REFERENCES}

1. C.L. Hill and X. Zhang, Nature, 373, 324 (1995).

2. K. Fukaya and T. Yamase, Angew. Chem. Int. Ed., 115, 678 (2003).

3. P.C. Li, S.H. Ye, S.H. Chen, J.P. Xiong, L. Wang and Y.H. Song, Asian J. Chem., 25, 1420 (2013).

4. F. Yao, Y.-G. Chen, A.R. Salimi and M. Mirzaei, J. Clust. Sci., 22, 309 (2011).

5. T.H. Li, J. Lu, S.Y. Gao and R. Cao, Inorg. Chem. Commun., 10, 551 (2007).

6. Q.G. Zhai, X.Y. Wu, S.M. Chen, L.J. Chen and C.Z. Lu, Inorg. Chim. Acta, 360, 3484 (2007) 